Related content

\section{Nanospintronic properties of carbon-cobalt atomic chains}

To cite this article: E. Durgun et al 2006 EPL 73642

View the article online for updates and enhancements. edpsciences IOP Institute of Physics

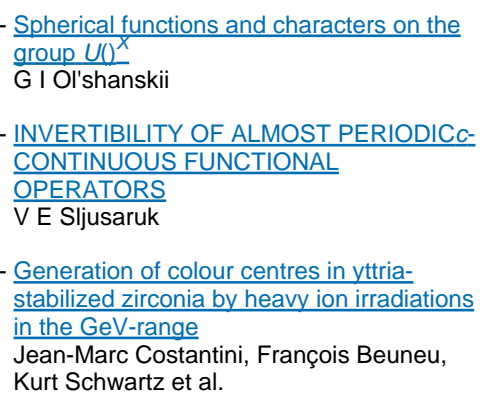

Spherical functions and characters on the group $U()^{x}$

G I Ol'shanskii

- INVERTIBILITY OF ALMOST PERIODICCCONTINUOUS FUNCTIONAL

OPERATORS

V E Sljusaruk

Generation of colour centres in yttriastabilized zirconia by heavy ion irradiations in the GeV-range

Jean-Marc Costantini, François Beuneu,

Kurt Schwartz et al.

\section{Recent citations}

- Structure-dependent metal-insulator
$\frac{\text { transition in one-dimensional Hubbard }}{\text { Superlattice }}$
Zhang Liang-Liang et al
- Contrasting magnetism in dilute and
$\frac{\text { supersaturated cobalt-fullerene mixture }}{\text { films }}$
V Lavrentiev et al
- Emergence of half-metallicity in
$\underline{\text { suspended NiO chains: Ab initio electronic }}$
$\frac{\text { structure and quantum transport }}{\text { calculations }}$
J. Fernández-Rossier et al


Europhys. Lett., 73 (4), pp. 642-648 (2006)

DOI: $10.1209 / \mathrm{epl} / \mathrm{i} 2005-10432-4$

\title{
Nanospintronic properties of carbon-cobalt atomic chains
}

\author{
E. Durgun ${ }^{1}$, R. T. Senger ${ }^{1}$, H. Mehrez ${ }^{2}$, S. Dag ${ }^{1}$ and S. Ciraci ${ }^{1}$ \\ 1 Department of Physics, Bilkent University - Bilkent, Ankara 06800, Turkey \\ 2 Center for NanoTechnology, NASA Ames Research Center \\ Moffett Field, CA 94035-1000, USA
}

received 8 November 2005; accepted 21 December 2005

published online 11 January 2006

PACS. 85.75.-d - Magnetoelectronics; spintronics: devices exploiting spin polarized transport or integrated magnetic fields.

PACS. 75.75. +a - Magnetic properties of nanostructures.

PACS. 75.47.Jn - Ballistic magnetoresistance.

\begin{abstract}
Periodic atom chains of carbon-cobalt compounds, $\left(\mathrm{C}_{n} \mathrm{Co}\right)_{\infty}$, comprise both conducting and insulating electronic properties simultaneously depending on the spin type of electrons, and hence are half-metals. Their band gap and the net magnetic moment oscillate with the number of carbon atoms in a unit cell. Finite segments of these chains also show interesting magnetic and transport properties. When connected to appropriate metallic electrodes the antiferromagnetic $\mathrm{CoC}_{n}$ Co segments behave like molecular spin-valves, which can be conveniently manipulated.
\end{abstract}

In earlier electronic transport studies, the spin-direction of conduction electrons was generally disregarded, in spite of the fact that spin orientation of electrons decays much slower than their momentum and consequently spin-coherence can be maintained relatively easily [1]. Current revolutionary applications using giant magnetoresistance in magnetic recording and nonvolatile memories have been derived from spin-dependent electronic transport (spintronics) [1-3]. Half-metals (HM) are a class of materials which exhibit interesting spintronics properties where electrons distinguished by their different spin orientation can assume different functions in the operation of the same device. This way, not only the capacity of information processing is doubled, but also new functions appear for important technological applications $[4,5]$. In HMs a $100 \%$ spin polarization is achieved, since the Fermi level $\left(E_{F}\right)$ is populated by states having only one spin direction but the empty states of the electrons of opposite spin are separated from the occupied states by an energy gap $E_{g}$. This situation is in contrast to the ferromagnetic metals, where both spins contribute to the state density at $E_{F}$ and spin polarization becomes less than 100\%. Even though 3D ferromagnetic Heusler alloys and transition metal oxides exhibit half-metallic properties [6], their stoichiometry cannot be controlled easily and coherent spin transport is destroyed by the defect levels. HMs such as $\mathrm{CrAs}$ and $\mathrm{CrSb}$ in zincblende structure have been grown only in thin-film forms [7].

In this letter we predict that periodic atom chains of carbon-cobalt compounds, $\left(\mathrm{C}_{n} \mathrm{Co}\right)_{\infty}$, comprise HM properties. Owing to the broken spin degeneracy energy bands split; the bands of one spin direction become semiconducting, but bands of the opposite direction show a 
metallic character. This way, the difference between the numbers of electrons of different spin orientations in the unit cell, $N=N_{\uparrow}-N_{\downarrow}$, is an integer and hence the spins become fully polarized at the Fermi level. In $\left(\mathrm{C}_{n} \mathrm{Co}\right)_{\infty}$ chains the band gap and the net magnetic moment show interesting even-odd disparity depending on the number of carbon atoms in a unit cell. In contrast to those complications found in three-dimensional (3D) structures [8], half-metallicity of $\left(\mathrm{C}_{n} \mathrm{Co}\right)_{\infty}$ chains appear to be rather robust and straightforward. Moreover, the number of carbon atoms in a unit cell provides a structural parameter to engineer the electronic and magnetic properties of these atomic chains, such as their band gap and magnetic moment. Similar half-metallic properties were also recently predicted for $\left(\mathrm{C}_{n} \mathrm{Cr}\right)_{\infty}$ chains [9]. While present $\left(\mathrm{C}_{n} \mathrm{Co}\right)_{\infty}$ compounds are metallic for minority spin states and semiconducting for majority spin states when $n>1$, in $\left(\mathrm{C}_{n} \mathrm{Cr}\right)_{\infty}$ compounds the character of majority and minority states alternate between metallic and semiconducting depending on $n$ being odd or even. The compound chains of other transition metal atoms, such as $\mathrm{Ti}, \mathrm{Fe}$, and $\mathrm{Mn}$, do not show half-metallic properties for all $n$.

Finite segments of carbon-cobalt chains show even more interesting properties. The magnetic ground state of the finite $\mathrm{CoC}_{n}$ Co chain structures is ferromagnetic for even- $n$, but antiferromagnetic for odd- $n$. When connected to appropriate metallic electrodes the antiferromagnetic $\mathrm{CoC}_{n}$ Co segments behave like molecular spin valves, which can be conveniently manipulated. We show that these compound atom chains are stable up to $1000 \mathrm{~K}$ and robust against axial elastic deformation. Their predicted properties are unusual for one-dimensional matter and of fundamental interest in the field of fermionic excitations with spin degree of freedom.

Carbon atom strings $\mathrm{C}_{n}$, which are precursor to $\mathrm{C}_{n}$ Co compounds, have been investigated for decades [10]. Finite segments of $\mathrm{C}_{n}$ have already been synthesized [11]. As an ultimate one-dimensional structure having only one atom in the cross-section, carbon strings can form only linear atomic chains and are stabilized by double bonds, which consist of a $\sigma$-bond of $2 s+2 p_{z}$ atomic orbitals along the chain axis and $\pi$-bonds of $2 p_{x}$ and $2 p_{y}$ orbitals. Because of cylindrical symmetry of the chain structure, the latter $\pi$-orbitals form a doubly degenerate but half-filled band, which crosses the Fermi level. The double-bond structure underlies the unusual properties of $\mathrm{C}_{n}$, such as its high axial strength, transversal flexibility and strong cohesion. For example, the elastic stiffness of $\mathrm{C}_{n}$, i.e., the second derivative of the strain energy per atom with respect to the axial strain, $\mathrm{d}^{2} E / \mathrm{d} \varepsilon^{2}$, was calculated to be $119 \mathrm{eV}$, which is twice the typical value for carbon nanotubes. Despite its low coordination number of two as compared to four in diamond or three in graphite, the cohesive energy of $\mathrm{C}_{n}$ is as large as $90 \%$ of that of diamond [10].

Mechanical, electronic and magnetic properties of $\mathrm{C}_{n}$ Co compounds are derived from those of $\mathrm{C}_{n}$ strings. Now our prime concern is to demonstrate that they are stable and they can, in fact, be synthesized without major difficulty. To this end, we carry out a state-of-the art, firstprinciples analysis based on quantum mechanics within density functional theory (DFT) [12] at zero and high temperatures [13]. Together with generalized gradient approximation, we used ultrasoft pseudopotentials and plane waves with cut-off energy of $350 \mathrm{eV}$. The finite and infinite atomic chains have been treated in supercell geometries using a tetragonal cell with lattice parameters of $a_{s c}=b_{s c}=10 \AA$ and $c_{s c}=c$, where $c$ is the lattice parameter of the chain. For finite chains an additional $10 \AA$ vacuum space in the axial direction is left in the supercell. Brillouin zone is sampled by 25 -special $\boldsymbol{k}$-points for the CCo chain. The number of $\boldsymbol{k}$-points is scaled according to the size of the unit cell for other periodic chains. Atomic positions (hence the geometric structure) and the lattice parameter $c$ are optimized by minimizing the total energy, as well as Hellmann-Feynman forces on the atoms and the strain. The convergence criteria adopted for the total energy and atomic forces are $10^{-5} \mathrm{eV}$ and $10^{-3} \mathrm{eV} / \AA$, respectively. The calculations for finite chains are also compared and 
TABLE I - Summary of results of spin-polarized first-principles calculations for $\left(\mathrm{C}_{n} \mathrm{Co}\right)_{\infty}(1 \leq n \leq 6)$ atomic chains. $\Delta E_{T}$ is the difference between spin-paired (non-magnetic) and spin-unpaired (magnetic) total energies; $\Delta E_{T}>0$ indicates that the compound has a magnetic ground state. $c$ is the optimized $1 D$ lattice parameter. $\mu$ is the net magnetic moment per unit cell. In the last two columns, $\mathrm{M}$ denotes the metallic character, and the numerals are the band gaps in $\mathrm{eV}$ for semiconducting (or insulating) band structures.

\begin{tabular}{lccccc}
\hline Structure & $\Delta E_{T}(\mathrm{eV})$ & $c(\AA)$ & $\mu\left(\mu_{B}\right)$ & Majority spin $(\uparrow)$ & Minority spin $(\downarrow)$ \\
\hline$(\mathrm{CCo})_{\infty}$ & 0.11 & 3.04 & 1.0 & $\mathrm{M}$ & 0.70 \\
$\left(\mathrm{C}_{2} \mathrm{Co}\right)_{\infty}$ & 1.22 & 4.96 & 3.0 & 3.70 & $\mathrm{M}$ \\
$\left(\mathrm{C}_{3} \mathrm{Co}\right)_{\infty}$ & 0.71 & 6.18 & 1.0 & 1.52 & $\mathrm{M}$ \\
$\left(\mathrm{C}_{4} \mathrm{Co}\right)_{\infty}$ & 0.99 & 7.52 & 3.0 & 2.80 & $\mathrm{M}$ \\
$\left(\mathrm{C}_{5} \mathrm{Co}\right)_{\infty}$ & 1.20 & 8.76 & 1.0 & 1.81 & $\mathrm{M}$ \\
$\left(\mathrm{C}_{6} \mathrm{Co}\right)_{\infty}$ & 1.02 & 10.1 & 3.0 & 2.29 & $\mathrm{M}$ \\
\hline
\end{tabular}

confirmed with those of GAUSSIAN 03 code [14] which utilizes local basis sets instead of plane waves. B3LYP and BPW91 DFT parametrization with cep31-g basis sets have been used. The reliability of GAUSSIAN 03 results have been further tested by performing wave function stability analysis. It is well documented that these calculations have been extremely successful in predicting novel nanostructures, such as carbon nanotubes, monatomic chains of carbon and transition metal atoms, even before these structures were synthesized [15]. Since all the atomic positions and the lattice parameter $c$ along the chain axis are optimized by minimizing the total energy of the whole system at $T=0 \mathrm{~K}$, as well as the forces on the atoms and the stress, the predicted geometric structures have a close bearing to the actual systems.

Transition state analysis performed for different reaction paths provides us with conclusive evidences showing that the linear $\mathrm{C}_{n}$ Co chains are stable and can be synthesized. The $\mathrm{C}_{n} \mathrm{Co}$ compound chains can conveniently grow from a finite $\mathrm{C}_{n}$ chain by attaching first $\mathrm{Co}$ and then $n \mathrm{C}$ atoms sequentially to one of its free ends, where individual Co and $\mathrm{C}$ atoms are attracted to their equilibrium bound state positions. No energy barrier is involved in the course of the growth and hence the process is exothermic. The minimum energy barrier to remove one Co atom from the $\left(\mathrm{C}_{n} \mathrm{Co}\right)_{\infty}$ chain in the transverse direction is calculated to be $1.1 \mathrm{eV}$, that is significantly high and renders stability. The energetics of the growth clearly demonstrates that $\mathrm{C}_{n}$ Co linear compounds are not simply a theoretical construct of fundamental interest, but they can also be realized experimentally. In fact, stable $\mathrm{C}_{n} \mathrm{Co}$ atom chains can be fabricated starting from one end of the finite-length $\mathrm{C}_{n}$ string which has been found at the center of a carbon nanotube [11]. In that geometry the carbon nanotube itself encapsulates the compound and protects it from oxidation and chemisorption of foreign atoms. Atomic manipulations using atomic force microscopy can be proposed as a potential technique for the fabrication of the prototypes of $\mathrm{C}_{n} \mathrm{Co}$ atom chains.

While first-principles total energy calculations at $T=0 \mathrm{~K}$ determines optimized structures by the conjugate gradient method, the stability of a $\left(\mathrm{C}_{n} \mathrm{Co}\right)_{\infty}$ compound is assured by stringent tests. For example, if a $\left(\mathrm{C}_{n} \mathrm{Co}\right)_{\infty}$ linear compound is stable, it corresponds to a local minimum of energy in the configuration space and this minimum shall be separated from other lower-energy local minima (or the global minimum) by sizable energy barriers. Such a case is examined first by displacing individual atoms of the compounds along various directions and then by allowing them to relax. In the present case all displaced atoms of $\left(\mathrm{C}_{n} \mathrm{Co}\right)_{\infty}$ have returned to their original configuration upon relaxation. A similar process is carried out by $a b$ initio molecular-dynamics calculations performed at high temperatures where individual atoms are moved in random directions resulting in sizable displacements. If the structure 

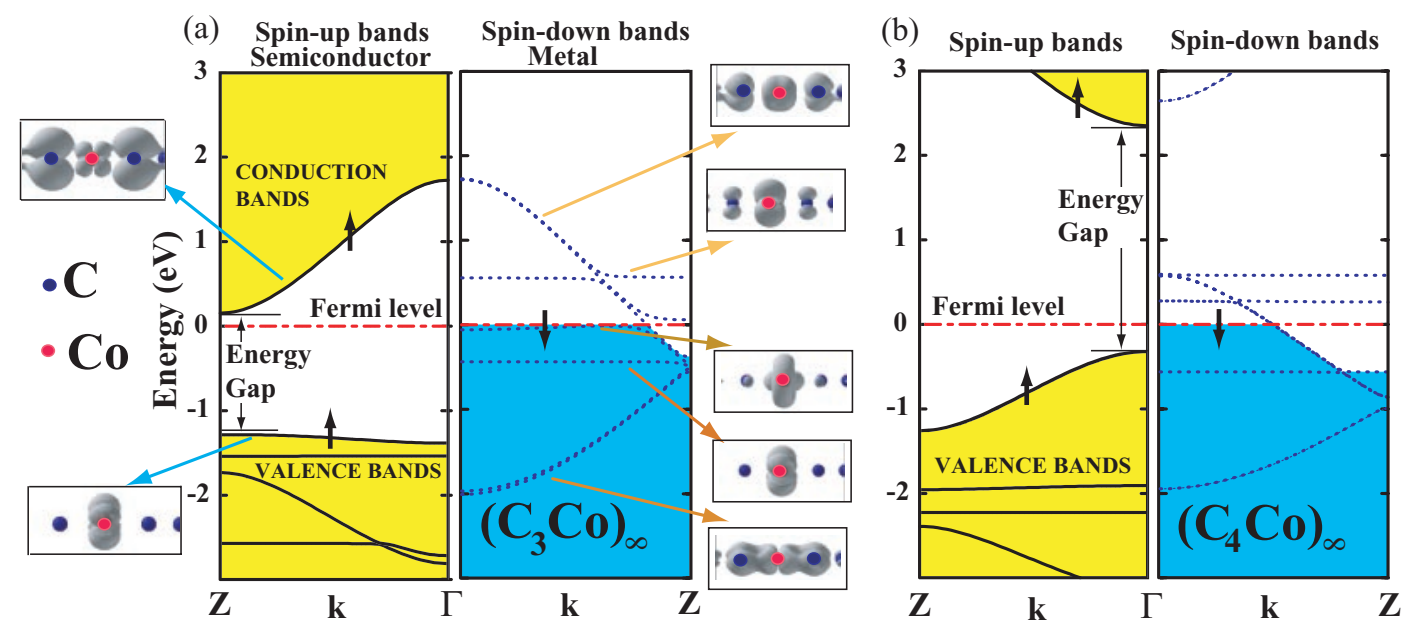

Fig. 1 - Energy band structures and charge densities of selected states. (a) $\left(\mathrm{C}_{3} \mathrm{Co}\right)_{\infty}$ compound. Semiconducting majority spin $(\uparrow)$ bands are shown in the left panel by continuous lines. The metallic minority spin $(\downarrow)$ bands are presented in the right panel by dotted lines along the $\Gamma$-Z. Electron charge density plots of selected states are shown in small side-panels. The lobs of Co- $3 d$ and C- $2 p$ orbitals are clearly seen. (b) Same for the $\left(\mathrm{C}_{4} \mathrm{Co}\right)_{\infty}$ linear chain.

under study were unstable or in a shallow local minimum, structural transformations could have taken place after a certain number of temperature-induced displacements. All these tests indicate that $\left(\mathrm{C}_{n} \mathrm{Co}\right)_{\infty}$ compounds $(1 \leq n \leq 6)$ shown in table I are stable. Higher $n$ values lead to instabilities and localization of the Co-3d states.

Spin-polarized electronic band structures of the $\left(\mathrm{C}_{n} \mathrm{Co}\right)_{\infty}$ compound chains in table I are half-metallic. These compounds show an even-odd $n$ disparity in electronic and magnetic properties. The compound $(\mathrm{CCo})_{\infty}$ (i.e. $\left.n=1\right)$ displays a wide angle zigzag geometry in equilibrium, and its minority-spin bands are semiconducting with a band gap $E_{g}=0.7 \mathrm{eV}$. In contrast, all $\left(\mathrm{C}_{n} \mathrm{Co}\right)_{\infty}(2 \leq n \leq 6)$ compounds are linear and are metal for minority spin bands but semiconductor for majority spin bands. The band gap is strongly dependent on $n$; for even $n, E_{g}$ is relatively large but decreases as $n$ increases. This situation is just the opposite for odd $n$. Moreover, the net magnetic moment per unit cell also exhibits an interesting even-odd $n$ disparity: All $\left(\mathrm{C}_{n} \mathrm{Co}\right)_{\infty}$ compounds have $\mu=1 \mu_{B}$ ( $\mu_{B}$ is Bohr magneton) for odd $n$, but $\mu=3 \mu_{B}$ for even $n$. While the spins on the $\mathrm{C}$ atoms adjacent to a Co atom are aligned along the same direction with that of the Co for even $n$, their spin direction is reversed when $n$ is odd. This latter situation is reminiscent of a ferrimagnetic order, in which Co and C sublattices carry opposite spins to reduce the net magnetic moment. The said variation of the magnetic moment with $n$ occurs because the Co-C bond distance varies according to $n$ being even or odd and hence changes the sign of the exchange integral $J$. Since the difference between spin-unpolarized (su) and spin-relaxed (sr) total energies, $\Delta E_{T}=E_{T}^{\mathrm{su}}-E_{T}^{\mathrm{sr}}$, and the net magnetic moment $\mu$ are positive, these compound chains have a ferromagnetic ground state. The spin-relaxed structure optimization starting with an initial net spin $S_{T}=0$ converged to the values $S_{T}=$ $\sum_{n, \boldsymbol{k}}^{\text {occ }} S_{n, \boldsymbol{k}}^{\uparrow}-S_{n, \boldsymbol{k}}^{\downarrow}$, so that an antiferromagnetic ground state is excluded for $\left(\mathrm{C}_{n} \mathrm{Co}\right)_{\infty}$ chains.

Half-metallicity of the $\left(\mathrm{C}_{3} \mathrm{Co}\right)_{\infty}$ and $\left(\mathrm{C}_{4} \mathrm{Co}\right)_{\infty}$ compounds is depicted by their band structure in fig. 1. While their majority spin bands are semiconducting, the minority spin bands are metallic with an equilibrium ballistic conductance of $G^{\downarrow}=e^{2} / h$ for $n=3$, and $G^{\downarrow}=2 e^{2} / h$ 


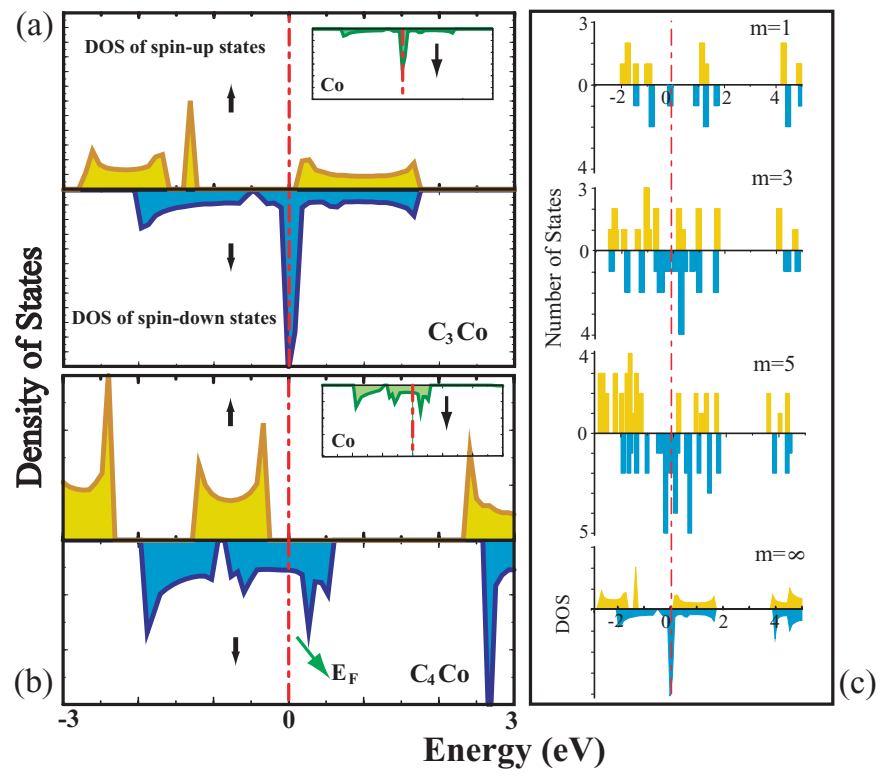

Fig. 2 - Total density of majority $(\uparrow)$ and minority $(\downarrow)$ spin states in arbitrary units. (a) $\left(\mathrm{C}_{3} \mathrm{Co}\right)_{\infty}$ compound. The density (DOS) of the majority spin states has a band gap and the Fermi level is close to the conduction band edge. The density of minority spin states is high at the Fermi level. These states are derived from the Co- $3 d$ and C- $2 p$ orbitals. (b) Same for the $\left(\mathrm{C}_{4} \mathrm{Co}\right)_{\infty}$ compound. The insets show that the metallic states at $E_{F}$ are derived from the $3 d$ states of Co atom. (c) DOSs calculated for $\left(\mathrm{C}_{3} \mathrm{Co}\right)_{m}$ chains show how the electronic structure of the infinite-periodic chain $(m=\infty)$ develops from those of the finite-length chains (i.e. $m=1,3,5)$ as $m$ increases.

for $n=4$. The number of $\mathrm{C}$ atoms in the unit cell determines whether the majority bands are an $n$-type or $p$-type semiconductor. The band gap is indirect for $n=3$ and exhibits an $n$-type character, but it is direct and $p$-type when $n=4$. The electronic charge density calculated for various states near the Fermi level indicates the dominant role of Co-3d orbitals in the electronic and magnetic properties of these compounds. The dispersive bands show significant Co- $3 d$ and C- $2 p$ hybridization. The band structure and charge density plots suggest that the $p$ - $d$ hybridization between neighboring $\mathrm{C}$ and Co orbitals and resulting exchange splitting of the bands corresponding to different spin directions give rise to a ferromagnetic ground state. The total density of spin states in fig. 2 illustrates the band gap of majority spin bands and a high density of minority spin states at $E_{F}$. Accordingly, the spin states at $E_{F}$ are $100 \%$ polarized and are derived mainly from Co-3d orbitals. Although small modifications to the band structure can be expected due to many-body effects, the band structure leading to a half-metallic character is found to be robust and is not affected by the axial tensile strain of $\varepsilon<0.05$. The spin-orbit coupling energy is also small and cannot influence the half-metallicity. Our calculation performed in a double unit cell demonstrated that the Peierls instability that may cause the splitting of the half-filled metallic bands at the Fermi level did not occur in the present system. The band picture depicted in fig. 1 ceases in small segments of $\left(\mathrm{C}_{n} \mathrm{Co}\right)_{m}$ due to broken translational symmetry and is replaced by the distribution of discrete states. However, as $m$ increases the continuous state distribution of $\left(\mathrm{C}_{n} \mathrm{Co}\right)_{\infty}$ is recovered and the weight of states localized at both ends of the chains is decreased (see fig. 2c).

Not only periodic atom chains but also finite linear segments or coils wound over a cylin- 


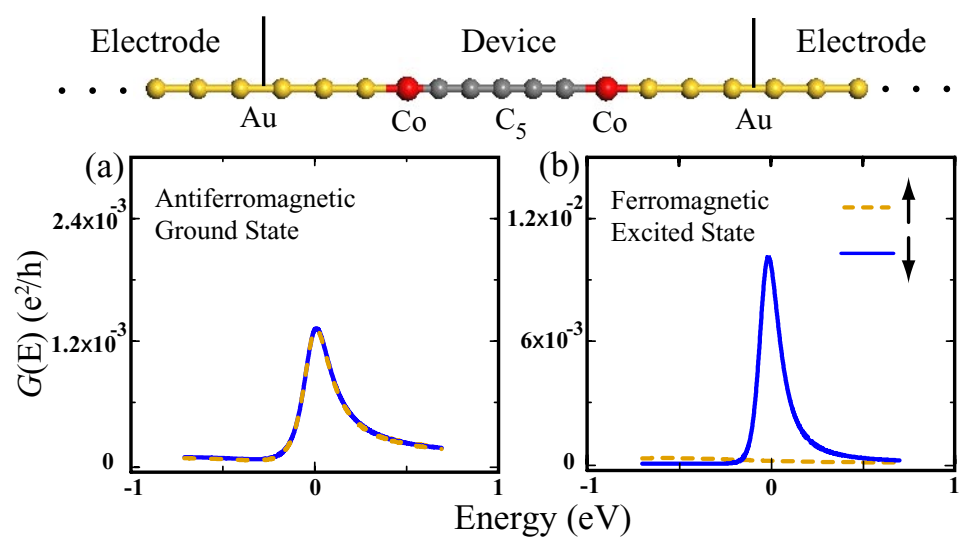

Fig. 3 - Spin-polarized ballistic conductance of $\mathrm{CoC}_{5} \mathrm{Co}$ chain placed between two gold electrodes: (a) Conductance $G$ for spin-up $(\uparrow)$ and spin-down $(\downarrow)$ electrons in the antiferromagnetic ground state. (b) Same for the ferromagnetic excited state. An order of magnitude enhanced conductance for spin-down, and almost vanishing value for the spin-up electrons indicate a strong spin-valve effect.

der can exhibit high spin-polarized electronic structure and offer properties leading to spinresonant tunneling and spin valve effects. Linear finite $\mathrm{CoC}_{n} \mathrm{Co}$ chains are also stable, but unlike the $\left(\mathrm{C}_{n} \mathrm{Co}\right)_{\infty}$ chains they have ferromagnetic ground state only for even- $n$. Conversely, for odd- $n$ they have antiferromagnetic ground state and ferromagnetic excited states. Their magnetic ground state alternates between ferromagnetic and antiferromagnetic configurations as $n$ is varied. The long-range indirect exchange coupling between the two Co atoms, which is mediated by the conduction electrons of carbon atoms [16], underlies the even-odd $n$ disparity and leads to a strong spin-valve effect for spintronic applications. Interestingly, in hybrid structures containing more than two Co atoms, such as $\mathrm{CoC}_{3} \mathrm{CoC}_{2} \mathrm{Co}$ and $\mathrm{CoC}_{3} \mathrm{CoC}_{3} \mathrm{Co}$, we find that the ground states are ferromagnetic with all the Co atoms having aligned local magnetic moments.

The transport characteristics of the finite $\mathrm{CoC}_{n}$ Co chains are calculated within Landauer formalism and Green's function method by attaching gold electrodes to both ends. We model the electrodes by simple monatomic gold chains, which are metallic. The device-electrode interface through head-on connection is correctly represented by the present electrodes. Relatively higher density of states of a more realistic electrode (a thicker wire) may increase the coupling between the device and electrodes; however, this would only lead to scaling the transmission coefficient curves by a factor. The shapes as well as the ratio of spin-up to spindown transmissions will persist. After all, the interconnects between the molecular devices are desired to be as thin as possible. First, the magnetic ground state of the $\mathrm{Au}_{6}-\mathrm{CoC}_{5} \mathrm{Co}-$ $\mathrm{Au}_{6}$ is confirmed to be antiferromagnetic, by solving the whole system in both ferromagnetic and antiferromagnetic states. The self-consistent calculations lead to Hamiltonian matrices of the device for the spin-up and spin-down electrons which are used to calculate the transmission coefficients for each spin state separately in the ground and excited magnetic states of the system. The conductance of the device is calculated using the Landauer formula $G(E)=\left(e^{2} / h\right) \operatorname{Tr}\left(\boldsymbol{\Gamma}_{\mathrm{L}} \boldsymbol{G}^{\mathrm{r}} \boldsymbol{\Gamma}_{\mathrm{R}} \boldsymbol{G}^{\mathrm{a}}\right)$ in terms of the retarded and advanced Green's functions, and the coupling matrices which are related to the recursively calculated self-energies of the semi-infinite gold electrodes.

In fig. 3 one can see that for the antiferromagnetic ground state of $\mathrm{CoC}_{5} \mathrm{Co}$ the conductance $G$ of spin-up electrons coincides with that of the spin-down electrons (i.e. spin valve is off). 
However, in a weak external magnetic field the system can be excited to its ferromagnetic state (with $\mu=4 \mu_{B}$ ), where $G$ of spin-down electrons is increased by an order of magnitude, but $G$ of spin-down electrons is substantially suppressed (i.e. spin valve is on). This is a clear indication of spin valve effect through the linear $\mathrm{CoC}_{5} \mathrm{Co}$ molecule between two metal electrodes. Earlier we have showed that carbon atomic strings can form stable 2D networks and $3 \mathrm{D}$ grid structures [10]. Since $\mathrm{CoC}_{n} \mathrm{Co}$, as well as $\mathrm{C}_{n} \mathrm{CoC}_{n}$ finite segments have magnetic ground states with significant net magnetic moment and can be attached to various parts of the carbon network or grid structure, they can provide high-density magnetic data storage medium such as magnetic RAMs. Clearly, half-metallic atom chains $\left(\mathrm{C}_{n} \mathrm{Co}\right)_{\infty}$ or their finite segments present a number of exciting properties, which are strongly dependent on the number of carbon atoms. These properties are of fundamental and technological interest and can initiate new concepts for spintronic devices and high-density data storage.

$$
* * *
$$

SC acknowledges helpful discussions with Prof. W. Kohn. We thank Prof. D. M. LeitneR and Dr M. BAYINDIR for their critical reading of the manuscript.

\section{REFERENCES}

[1] Wolf S. A., Awschalom D. D., Buhrman R. A., Daughton J. M., von Molnar S., Roukes M. L., Chtchelkanova A. Y. and Treger D. M., Science, 294 (2001) 1488.

[2] Prinz G. A., Science, 282 (1988) 1660.

[3] Ball P., Nature, 404 (2000) 918.

[4] De Groot R. A., Mueller F. M., van Engen P. G. and Buschow K. H. J., Phys. Rev. Lett., 50 (1983) 2024.

[5] Pickett W. E. and Moodera J. S., Phys. Today, 54 (2001) 39.

[6] Park J. H., Vescovo E., Kim H. J., Kwon C., Ramesh R. and Venkatesan T., Nature, $392(1998) 794$.

[7] Akiniga H., Manago T. and Shira M., Jpn. J. Appl. Phys. Part 2, 39 (2000) L1118.

[8] Pask J. E., Yang L. H., Fong C. Y., Pickett W. E. and Dag S., Phys. Rev. B, 67 (2003) 224420.

[9] Dag S., Tongay S., Yildirim T., Durgun E., Senger R. T., Fong C. Y. and Ciraci S., Phys. Rev. B, 72 (2005) 155444.

[10] Tongay S., Senger R. T., Dag S. and Ciraci S., Phys. Rev. Lett., 93 (2004) 136404; Tongay S., Dag S., Durgun E., Senger R. T. and Ciraci S., J. Phys. Condens. Matter, 17 (2005) 3823; Senger R. T., Tongay S., Durgun E. and Ciraci S., Phys. Rev. B, 71 (2005) 235406.

[11] Roth G. and Fischer H., Organometallics, 15 (1996) 5766; Zhao X., Ando Y., Liu Y., Jinno M. and Suzuki T., Phys. Rev. Lett., 90 (2003) 187401.

[12] Hohenberg P. and Kohn W., Phys. Rev., 136 (1964) B864; Kohn W. and Sham L. J., Phys. Rev., 140 (1965) A1133.

[13] Numerical calculations have been performed by using vaSP package: Kresse G. and HAFneR J., Phys. Rev. B, 47 (1993) 558; Kresse G. and Furthmüller J., Phys. Rev. B, 54 (1996) 11169.

[14] Gaussian 03, revision C.02, Frisch M. J. et al., Gaussian, Inc., Wallingford, CT, 2004.

[15] Ciraci S., Dag S., Yildirim T., Gulseren O. and Senger R. T., J. Phys. Condens. Matter, 16 (2004) R901.

[16] Costa A. T., Kirwan D. F. and Ferreira M. S., Phys. Rev B, 72 (2005) 085402. 